\title{
Chronic dialysis patients with infectious spondylodiscitis have poorer outcomes than non-dialysis populations
}

This article was published in the following Dove Press journal:

Therapeutics and Clinical Risk Management

\author{
George Kuo' \\ Wei-Chiao Sun' \\ Yueh-An Lu' \\ Chao-Yu Chen' \\ Huang-Kai Kao ${ }^{2}$ \\ YuJr $\operatorname{Lin}^{3}$ \\ Yung-Chang Chen ${ }^{4}$ \\ Cheng-Chieh Hung' \\ Ya-Chung Tian' \\ Hsiang-Hao Hsu' \\ 'Department of Nephrology, Kidney \\ Research Center, College of Medicine, \\ Linkou Chang Gung Memorial \\ Hospital, Chang Gung University, \\ Taoyuan, Taiwan; ${ }^{2}$ Department of \\ Plastic and Reconstructive Surgery, \\ College of Medicine, Linkou Chang \\ Gung Memorial Hospital, Chang Gung \\ University, Taoyuan, Taiwan; ${ }^{3}$ Center \\ for Big Data Analytics and Statistics, \\ College of Medicine, Linkou Chang \\ Gung Memorial Hospital, Chang \\ Gung University, Taoyuan, Taiwan; \\ ${ }^{4}$ Department of Internal Medicine, \\ Division of Nephrology, College \\ of Medicine, Keelung Chang Gung \\ Memorial Hospital, Chang Gung \\ University, Taoyuan, Taiwan
}

Correspondence: Hsiang-Hao Hsu Department of Nephrology, Kidney Research Center, Linkou Chang Gung Memorial Hospital, No 5 Fu-Shin Street, Kweishan, Taoyuan 333, Taiwan

Tel +886 3328 I 200 ext $8|8|$

Fax +88633282173

Email hsianghao@gmail.com
Purpose: Infectious spondylodiscitis is a serious disease that can lead to permanent neurological deficit. Because there were only a few case reports or series featuring infectious spondylodiscitis in chronic dialysis patients, we investigated the epidemiology and outcome in the chronic dialysis patients versus general population.

Materials and methods: We retrospectively identified chronic dialysis patients admitted for infectious spondylodiscitis between January 2002 and December 2015. A total of 105 chronic dialysis patients were included, and we performed a 1:2 case-control match on propensity score in non-dialysis patients with infectious spondylodiscitis. The demographic features, clinical manifestation, infection focus, and disease outcome were recorded.

Results: A total of 302 patients entered the final analysis. Chronic dialysis patients less frequently had fever (34.3\%), and in the majority, bacterial entry was through dialysis vascular access (30.5\%). Methicillin-resistant Staphylococcus aureus (MRSA) comprised the majority of causative pathogen. The chronic dialysis group had longer hospital stay, higher in-hospital mortality, and higher 1-year mortality. The odds ratio of in-hospital mortality was 2.20 compared with the non-dialysis group.

Conclusions: The study highlighted poorer outcome and high frequency of resistant Staphylococcus of infectious spondylodiscitis in chronic dialysis patients. Therefore, high vigilance, prompt recognition, and empiric coverage of MRSA will be important in the management of infectious spondylodiscitis in chronic dialysis patients.

Keywords: end stage renal disease, pyogenic spondylodiscitis, infectious spondylodiscitis, methicillin-resistant Staphylococcus aureus, mortality

\section{Introduction}

Infectious spondylodiscitis is a relatively uncommon but serious disease that can lead to permanent neurological deficit or chronic pain. It may be diagnosed late because of relative insidious onset, and non-specific symptoms. In recent years, the incidence of infectious spondylodiscitis seems to have increased with the development of advanced radiographic diagnosis, increased spinal intervention, and increased life expectancy. ${ }^{1}$ The microorganisms inoculate vertebra via several pathways. The hematogenous spread of pathogen from another primary focus remains the major mechanism. Retrograde, ascending infection from the urinary tract, direct invasion from surrounding tissues, or contamination during an invasive or surgical procedure are also possible contributing mechanisms. ${ }^{2-6}$

The patients with end-stage renal disease (ESRD), especially those on chronic maintenance hemodialysis, are susceptible to bloodstream infection because of infected 
vascular access, repetitive vascular puncture, or dialysis water purification system. The immune dysfunction under uremia milieu may also contribute to the inability to defend against microorganism invasion. ${ }^{7-10}$ With these additional risk factors of infection, the clinical presentation, microbiology characteristics, and the outcome may be different in the chronic dialysis population compared with the nondialysis counterpart. To date, there are only a few case reports and small series discussing infectious spondylodiscitis in the dialysis population. ${ }^{11-18}$ This study aims to elucidate the epidemiology, risk factors, and outcome in infectious spondylodiscitis in a larger chronic dialysis population.

\section{Materials and methods}

\section{Patient selection and data collection}

The study was carried out at a tertiary referral center with about 3,700 beds and an average of 10,700 episodes of in-patient service annually. This study was approved by the Institutional Review Board of Chang Gung Memorial Hospital (IRB No 201600819B0C101). The need for written informed consent was waived by the IRB because this study was retrospective, non-interventional design, and patient data confidentiality and privacy was maintained.

We searched the discharge diagnosis from the health information system in the study hospital by International Classification of Disease-9 (ICD-9) code (720.9: unspecified inflammatory spondylopathy) between January 2002 and August 2015. Patients discharged with ICD-9 720.9 and ESRD were included. We excluded patients who were aged $<20$ years, started dialysis therapy during the index hospitalization, underwent dialysis $<14$ days, received solid organ or hematopoietic stem cell transplantation, had recurrent infectious spondylodiscitis, or those treated incompletely owing to personal reasons. We performed a 1:2 case-control match on propensity score depending on age, sex, and the presence of diabetes mellitus (DM) from the non-dialysis group.

The patients' demographic information, clinical presentations, laboratory data, and survival were recorded by chart review. The serum C-reactive protein (CRP) level at baseline and 1 week after treatment were documented. The causative pathogens were determined if the microorganism grew from the blood, abscess, and/or tissue culture. The numbers and levels of the involved vertebrae were documented from image study, including CT, MRI, and/or the inflammatory scan with Gallium radio-isotope or ${ }^{18} \mathrm{~F}$-fluorodeoxyglucose (FDG).

\section{Statistical analysis}

The categorical variables are presented with proportion and compared by Chi-square test. The continuous variables are presented with mean and SD, and these variables are tested by independent $t$-test. Patient survival between groups was compared with Kaplan-Meier analysis. A 2-sided $p$-value $<0.05$ was considered to be statistically significant. The statistical analysis was done with SPSS version 17.

\section{Results}

Figure 1 illustrates the patient selection procedure. Between January 2002 and August 2015, we found 1,402 hospitalized patients discharged with the ICD-9 diagnosis code 720.9 . Among them, 106 patients were identified with a diagnosis of ESRD undergoing chronic dialysis. We performed a 1:2 case-control match on propensity score depending on age, sex, and the presence of DM. After thorough chart review, we excluded 7 cases admitted for recurrent infectious spondylodiscitis and 9 cases discharged against medical advice before completing treatment. A total of 302 patients entered the final analysis, with 105 patients in the chronic dialysis group and 197 patients in the non-dialysis control.

The demographics are shown in Table 1. The chronic dialysis group had significantly more hypertension, coronary artery disease, and congestive heart failure. The prevalence of degenerative spinal disease was lower in the chronic dialysis group. No statistic differences were found in the co-morbidities, including cirrhosis, active malignancy, use of immunosuppressive agents, or vertebral trauma.

Table 2 summarizes the clinical characteristics of infectious spondylodiscitis. Back pain was the major symptom in both groups, while fever occurred in less than half of both groups. The chronic dialysis patients had fever less frequently compared with non-dialysis patients (34.3\% vs $46.7 \%, p=0.038)$. Dialysis vascular access infection was shown to be a major focus (30.5\%) of bacterial entry in the chronic dialysis group. The initial laboratory data demonstrated higher blood urea nitrogen, creatinine, and lower hemoglobin in the chronic dialysis group. Both the baseline erythrocyte sedimentation rate and the 1-week post-treatment CRP were higher in the chronic dialysis group. No differences were found in the level of infected vertebrae and the frequency of skipped lesions, but the chronic dialysis patients had less abscess formation ( $48.6 \%$ vs $60.7 \%, p=0.039$ ).

Table 3 summarizes the pathogen spectrum in both groups. The Gram-positive cocci (GPC) were isolated more frequently in the chronic dialysis group $(59.1 \%$ vs $42.6 \%$, $p=0.0064)$. Methicillin-resistant Staphylococcus aureus (MRSA) comprised the majority of GPC isolates from dialysis patients $(28.6 \%$ vs $11.2 \%, p<0.0001)$. The Gramnegative bacilli (GNB) were rare in the chronic dialysis group $(0.95 \%$ vs $14.7 \%, p=0.0001)$. The frequency of fungal, 


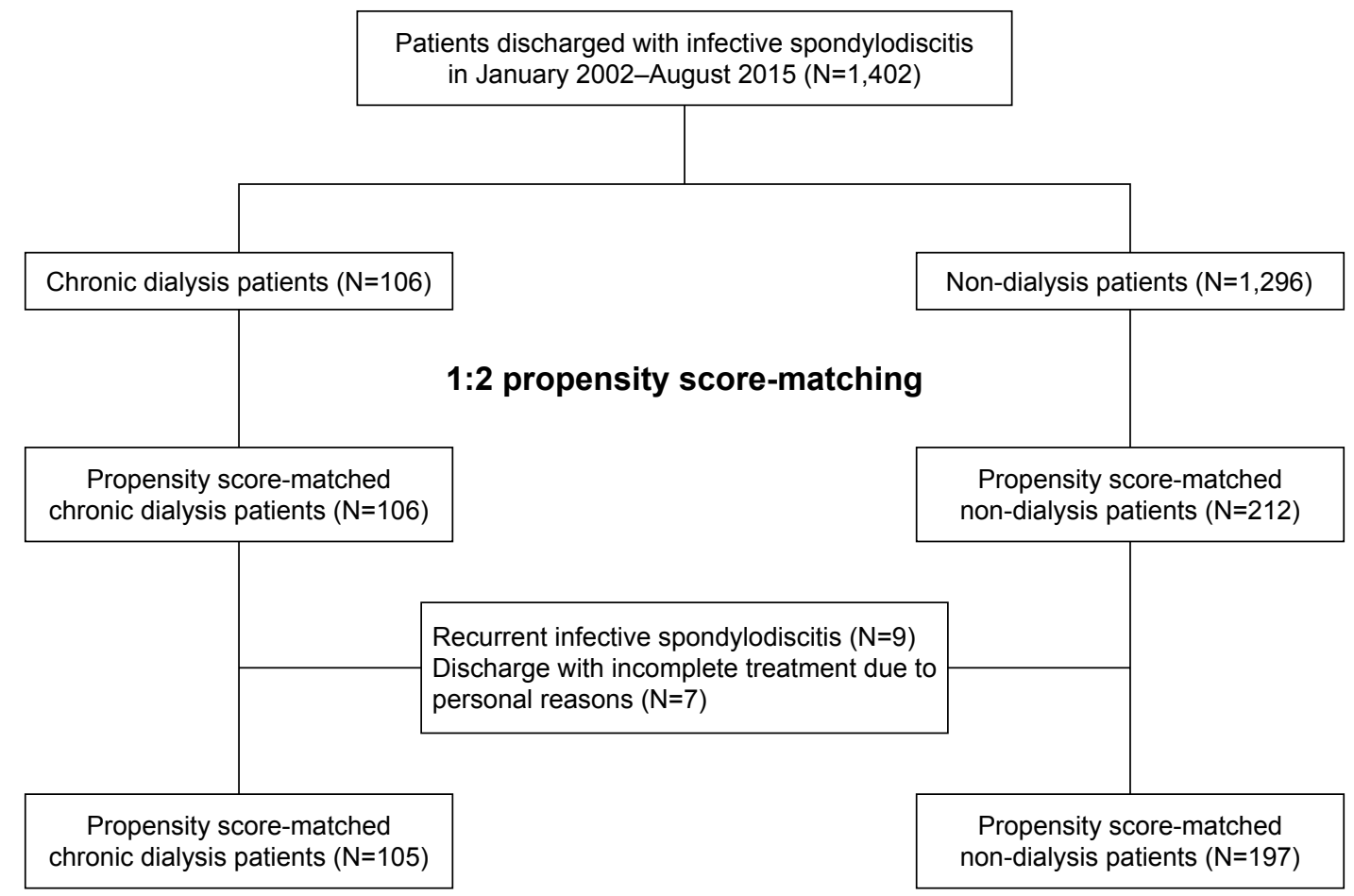

Figure I The flowchart of study patient enrollment.

mycobacterial, or polymicrobial infectious spondylodiscitis were similarly rare in both groups.

Out of the total number of patients, 37 died in the hospital, only 2 died of causes other than sepsis (one from acute myocardial infarction and the other one from massive gastrointestinal [GI] bleeding). Another 9 patients who survived at discharge, died within 1 year. The causes of death included 1 GI bleeding, 1 severe aortic stenosis with refractory heart failure, 2 with recurrent spondylodiscitis, and 5 due to pneumonia. With respect to outcome, the chronic dialysis group had longer hospital stay and worse in-hospital survival (Table 4). The Kaplan-Meier survival analysis also demonstrated

Table I Demographics of chronic dialysis vs non-dialysis group

\begin{tabular}{|c|c|c|c|}
\hline Patient characteristics & $\begin{array}{l}\text { Chronic dialysis } \\
(\mathrm{N}=105)(\%)\end{array}$ & $\begin{array}{l}\text { Non-dialysis } \\
(\mathrm{N}=197)(\%)\end{array}$ & $p$-value \\
\hline Age (years), mean \pm SD & $66.7 \pm 10.8$ & $63.2 \pm 9.4$ & 0.977 \\
\hline Male gender & $51(48.6)$ & $96(48.7)$ & 0.979 \\
\hline Diabetes mellitus & $51(48.6)$ & $89(45.2)$ & 0.573 \\
\hline Hypertension & $67(63.8)$ & $55(27.9)$ & $<0.001$ \\
\hline Coronary artery disease & $23(21.9)$ & II (5.6) & $<0.001$ \\
\hline Congestive heart failure & $17(16.2)$ & $15(7.6)$ & 0.021 \\
\hline Cerebrovascular accident & $12(11.4)$ & $14(7.1)$ & 0.202 \\
\hline Cirrhosis & $10(9.5)$ & $20(10.2)$ & 0.862 \\
\hline Active malignancy & $6(5.7)$ & $14(7.1)$ & 0.643 \\
\hline Immunosuppression & $3(2.9)$ & $14(7.1)$ & 0.127 \\
\hline Degenerative spinal disease & $89(84.8)$ & I $86(94.4)$ & 0.005 \\
\hline Vertebral trauma & $3(2.9)$ & $15(7.6)$ & 0.094 \\
\hline
\end{tabular}

worse 1-year survival in the chronic dialysis group (Figure 2, $\log$-rank $p=0.004)$. The odds ratio of hospital death in chronic dialysis group was 2.20 (95\% CI: $1.10-4.40, p=0.026)$ compared with the non-dialysis control. The 1-year recurrence rates were the same in both groups.

\section{Discussion}

Infectious spondylodiscitis is an uncommon but devastating disease that can lead to permanent neurological deficit, chronic pain, and even mortality. Risk factors for infectious spondylodiscitis include diabetes, endocarditis, degenerative spine disease, prior spinal surgery, corticosteroid therapy, or other immunocompromised states. Its incidence has been increasing in the recent years, likely due to the following reasons: increasing rates of bacteremia due to intravascular devices and other forms of instrumentation; increasing age of the population; and increasing number of patients on renal replacement therapy. ${ }^{19}$

Till now, there have been no randomized trials evaluating outcome of infectious spondylodiscitis in ESRD patients with chronic dialysis, which is the most common type of renal replacement therapy. In this study, we performed a 1:2 case-control match on propensity score depending on age, sex, and the presence of DM to compare the epidemiology, risk factors, and outcome of infectious spondylodiscitis in chronic dialysis patients with the non-dialysis patients. 
Table 2 Clinical characteristics of infectious spondylodiscitis in chronic dialysis vs non-dialysis group

\begin{tabular}{|c|c|c|c|}
\hline Clinical features & $\begin{array}{l}\text { Chronic dialysis } \\
(\mathrm{N}=105)(\%)\end{array}$ & $\begin{array}{l}\text { Non-dialysis } \\
(\mathrm{N}=197)(\%)\end{array}$ & $p$-value \\
\hline \multicolumn{4}{|l|}{ Disease manifestations } \\
\hline Fever & $36(34.3)$ & $92(46.7)$ & 0.038 \\
\hline Back pain & $80(76.2)$ & $164(83.2)$ & 0.138 \\
\hline Shock & $24(22.9)$ & $33(16.8)$ & 0.197 \\
\hline $\begin{array}{l}\text { Concurrent infective } \\
\text { endocarditis }\end{array}$ & $2(1.9)$ & $4(2.0)$ & 0.941 \\
\hline \multicolumn{4}{|l|}{ Possible primary focus } \\
\hline Spine surgery & $9(8.6)$ & $26(13.2)$ & $<0.001$ \\
\hline Soft tissue infection & $5(4.8)$ & $13(6.6)$ & \\
\hline Vascular access & $32(30.5)$ & $0(0)$ & \\
\hline Pneumonia & $0(0)$ & $5(2.5)$ & \\
\hline Urinary tract infection & $2(1.9)$ & $17(8.6)$ & \\
\hline Peritonitis & $0(0)$ & $\mathrm{I}(0.5)$ & \\
\hline Unknown & $57(54.3)$ & $135(68.5)$ & \\
\hline Positive tissue culture & $39(37.1)$ & $84(42.6)$ & 0.238 \\
\hline Positive blood culture & $60(57.1)$ & $85(43.1)$ & 0.387 \\
\hline \multicolumn{4}{|l|}{ Laboratory data } \\
\hline Hemoglobin (g/dL) & $9.4 \pm 1.6$ & $10.7 \pm 2.1$ & $<0.001$ \\
\hline Albumin $(g / d L)$ & $3.0 \pm 0.5$ & $2.9 \pm 0.7$ & 0.384 \\
\hline $\begin{array}{l}\text { Blood urea nitrogen } \\
(\mathrm{mg} / \mathrm{dL})\end{array}$ & $48.7 \pm 23.6$ & $26.3 \pm 21.9$ & $<0.001$ \\
\hline Creatinine $(\mathrm{mg} / \mathrm{dL})$ & $6.2 \pm 2.3$ & $1.3 \pm 1.2$ & $<0.001$ \\
\hline Alkaline phosphatase (U/L) & $164.5 \pm 143.50$ & $142.6 \pm 93.2$ & 0.182 \\
\hline CRP (baseline) (mg/L) & $|23.7 \pm 9| .5$ & $122.9 \pm 113.9$ & 0.951 \\
\hline $\begin{array}{l}\text { CRP (I week after } \\
\text { treatment) (mg/L) }\end{array}$ & $85.1 \pm 71.8$ & $68.4 \pm 61.8$ & 0.046 \\
\hline $\begin{array}{l}\text { WBC (baseline) } \\
(1,000 / \mu \mathrm{L})\end{array}$ & $11.8 \pm 5.8$ & $11.8 \pm 6.6$ & 0.997 \\
\hline $\begin{array}{l}\text { WBC (I week after } \\
\text { treatment) }(I, 000 / u L)\end{array}$ & $10.1 \pm 4.4$ & $10.1 \pm 12.7$ & 0.140 \\
\hline $\operatorname{ESR}(\mathrm{mm} / \mathrm{hr})$ & $86.6 \pm 33.6$ & $70.0 \pm 31.7$ & 0.001 \\
\hline \multicolumn{4}{|c|}{ Features of the involved vertebral lesions } \\
\hline Cervical spine & $\mathrm{II}(10.5)$ & $21(10.7)$ & 0.961 \\
\hline Thoracic spine & $14(13.3)$ & $39(19.8)$ & 0.160 \\
\hline Lumbar spine & $89(84.8)$ & $150(76.1)$ & 0.079 \\
\hline Sacral spine & $10(9.5)$ & $27(\mid 3.7)$ & 0.291 \\
\hline Skipping lesions & $5(4.8)$ & $5(2.5)$ & 0.304 \\
\hline Abscess formation & $51(48.6)$ & $120(60.9)$ & 0.039 \\
\hline \multicolumn{4}{|l|}{ Invasive interventions } \\
\hline CT-guided drainage & $14(13.3)$ & $19(9.6)$ & 0.328 \\
\hline Surgery & $56(53.3)$ & $124(62.9)$ & 0.105 \\
\hline
\end{tabular}

Abbreviations: CRP, C-reactive protein; ESR, erythrocyte sedimentation rate; WBC, white blood cells.

Our study found the higher in-hospital and 1-year mortality rate in ESRD patients with infectious spondylodiscitis compared with non-dialysis population. This is in concordance with previous studies that have examined the outcome of other infections in chronic dialysis patients. Sarnak et al reported higher mortality in patients with ESRD who were hospitalized for sepsis or pneumonia. ${ }^{20,21}$ In ESRD patients diagnosed with infective endocarditis, smaller studies did not find differences in mortality; $;^{22,23}$ however, a larger nationwide study involving $\sim 250,000$ patients in the USA found a significantly higher in-hospital mortality in ESRD populations. ${ }^{24}$

Higher prevalence of hypertensive cardiovascular disease, coronary artery disease, and congestive heart failure among the ESRD patients may partly explain the increasing mortality in this population. Tonelli et al reported that patients with chronic kidney disease have a higher cardiovascular risk and as much as $58 \%$ of mortality may be attributed to cardiovascular events. ${ }^{25}$ In patients hospitalized for sepsis and septic shock, pre-existing cardiac diseases, including impaired systolic or diastolic left ventricular function were associated with higher mortality. ${ }^{26,27}$ In our study, the dialysis group had more pre-existing hypertension, coronary artery disease, and congestive heart failure. These co-morbidities, which impair cardiovascular adaptations in facing septic status, may contribute to the higher mortality observed in our chronic dialysis patients than the non-dialysis patients. Degenerative spine disease is less common in the chronic dialysis group. This may be contrary to the usual belief that degenerative spine disease and subsequent surgery increase the risk of infectious spondylodiscitis. ${ }^{19}$ A possible explanation of this difference may be that it resulted from the fact that some of the dialysis patients acquired spine infection from other sources, for example, repetitive bacteremia from vascular access.

Another significant difference highlighted by our study is the higher proportion of MRSA in the chronic dialysis group. Previous studies in infectious spondylodiscitis of general population demonstrated that GPC is the most common pathogen and followed by GNB. Methicillin-susceptible $S$. aureus (MSSA) remained the most common bacteria among GPC, while Escherichia coli accounts for up to onethird of the GNB group. ${ }^{1,6,28}$

Patients on chronic hemodialysis are associated with increased risk of Staphylococcal infection. Two nationwide studies from Denmark reported a high incidence of overall bloodstream infection (137 per 1,000 person-year vs 5.3 per 1,000 person-year) and Staphylococcal bacteremia (35.7 per 1,000 person-year vs 0.5 per 1,000 person-year) in dialysis group compared with general population. ${ }^{29,30} \mathrm{~S}$. aureus bacteremia accounts for up to $30 \%-40 \%$ of bloodstream infection in the hemodialysis group. ${ }^{31,32}$ The hemodialysis vascular access is one of the most important route for bacterial entry. Zhang et al reported organism-specific rate of bloodstream infection in patients using different dialysis vascular access. The study demonstrated lowest $S$. aureus bacteremia in those using native arterio-venous fistula. However, the rate is still higher than in general population. ${ }^{29,33}$ 
Table 3 Microbiologic data of the chronic dialysis vs non-dialysis group

\begin{tabular}{|c|c|c|c|}
\hline Microorganism species & $\begin{array}{l}\text { Chronic dialysis } \\
(\mathrm{N}=105)(\%)\end{array}$ & $\begin{array}{l}\text { Non-dialysis } \\
(\mathrm{N}=197)(\%)\end{array}$ & $p$-value \\
\hline Gram-positive cocci & $62(59.1)$ & $84(42.6)$ & 0.0064 \\
\hline Methicillin-susceptible Staphylococcus aureus & $9(8.6)$ & $38(19.2)$ & \\
\hline Methicillin-resistant S. aureus & $30(28.6)$ & $22(11.2)$ & \\
\hline Coagulase-negative staphylococci & $14(13.3)$ & $8(4.1)$ & \\
\hline Streptococcus spp. & $2(1.9)$ & $12(6.1)$ & \\
\hline Enterococcus & $7(6.6)$ & $4(2.0)$ & \\
\hline Gram-negative bacilli & I $(0.95)$ & $29(14.7)$ & 0.0001 \\
\hline Escherichia coli & $0(0)$ & $14(7.1)$ & \\
\hline Klebsiella pneumoniae & $\mathrm{I}(0.95)$ & $8(4.1)$ & \\
\hline Pseudomonas aeruginosa & $0(0)$ & $4(2.0)$ & \\
\hline Salmonella enterica & $0(0)$ & $3(1.5)$ & \\
\hline Fungus & I (0.95) & $4(2.0)$ & 0.4939 \\
\hline Candida parapsilosis & I (0.95) & $\mathrm{I}(0.5)$ & \\
\hline Candida glabrata & $0(0)$ & $\mathrm{I}(0.5)$ & \\
\hline Candida albicans & $0(0)$ & $\mathrm{I}(0.5)$ & \\
\hline Yeast-like & $0(0)$ & $\mathrm{I}(0.5)$ & \\
\hline Mycobacterium & $2(1.9)$ & $6(3.1)$ & 0.5395 \\
\hline Mycobacterium tuberculosis & I $(0.95)$ & $4(2.0)$ & \\
\hline Mycobacterium chelonae & I $(0.95)$ & $\mathrm{I}(0.5)$ & \\
\hline Mycobacterium abscessus & $0(0)$ & $\mathrm{I}(0.5)$ & \\
\hline Polymicrobial isolates & $4(3.8)$ & $7(3.6)$ & 0.9300 \\
\hline Others & I (0.95) & $5(2.5)$ & \\
\hline Unknown & $34(32.4)$ & $62(31.5)$ & 0.8731 \\
\hline
\end{tabular}

Patients with MRSA bacteremia had higher mortality compared with those with MSSA bacteremia. ${ }^{34}$ Despite the use of appropriate antibiotic, a significantly high mortality was found in the MRSA compared with MSSA infections. ${ }^{35}$

There is no literature directly indicating a low frequency of GNB infectious spondylodiscitis in the dialysis group. Kang et al reported that GNB accounted for $18 \%$ of the pathogen in a 344-patients' series. Multivariate analysis showed that female gender, co-existing urinary tract or intra-abdominal infection were strongly associated with the development of GNB infectious spondylodiscitis. ${ }^{2}$ From this point of view, the extremely low rate of co-existing urinary tract or intra-abdominal infection may indirectly explain the low occurrence of GNB in the dialysis group.

Uremia has been linked to immune dysfunction. The most important first-line defense toward bacterial infection, that is, the neutrophil and macrophage, is dysregulated. Both

Table 4 Outcomes of the chronic dialysis vs non-dialysis group

\begin{tabular}{llll}
\hline Outcomes & $\begin{array}{l}\text { Chronic dialysis } \\
(\mathbf{N}=105)(\%)\end{array}$ & $\begin{array}{l}\text { Non-dialysis } \\
(\mathbf{N}=197)(\%)\end{array}$ & P-value \\
\hline $\begin{array}{l}\text { Length of hospital stay } \\
\text { (days), mean } \pm \text { SD }\end{array}$ & $62.78 \pm 39.30$ & $51.65 \pm 30.43$ & 0.012 \\
In-hospital survival & $86(81.9)$ & $179(90.9)$ & 0.024 \\
I-year survival & $80(76.2)$ & $176(89.3)$ & 0.002 \\
I-year recurrence & I7 (I6.2) & $39(19.8)$ & 0.722 \\
\hline
\end{tabular}

neutrophil and macrophage have higher baseline expression of Toll-like receptors and synthesis of reactive oxygen species, but these cells present with impaired phagocytosis while encountering the pathogen..$^{7,9,10}$ The adaptive immune cells are also quantitatively and qualitatively impaired in

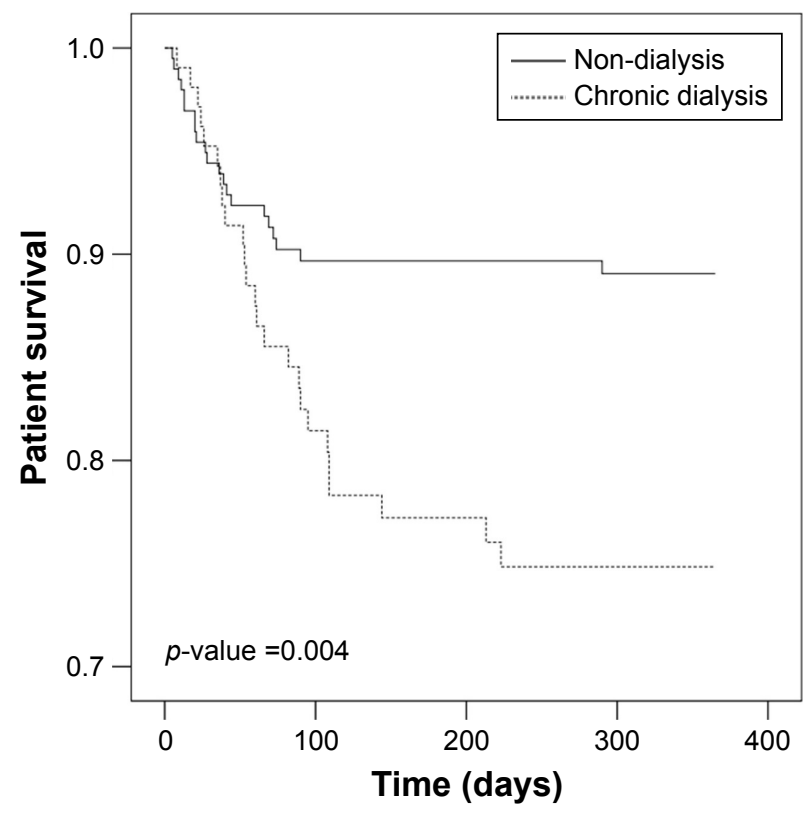

Figure 2 Kaplan-Meier survival curves of I-year survival between chronic dialysis and non-dialysis patients with infectious spondylodiscitis. 
the uremia milieu. ${ }^{7,8}$ However, these phenomena are mostly observed in vitro or ex vivo. There is lack of direct evidence of the aforementioned immune dysfunction on patient outcome. In our study, the dialysis group presented with less fever compared with non-dialysis control. A recent study in Korea found afebrile status during bacteremia was associated with mortality in chronic dialysis patients. Elevated CRP, on the other hand, remained a good indicator of bacterial infection. ${ }^{36}$ Although the direct link between immune dysfunction and less presentation with fever is not clear, this phenomenon reminds the clinician to be alert.

There are still limitations in this study. The study enrolled patients from single medical center and did not include patients with less severe disease. This may limit generalization of our results to a larger population. Second, the attribution of infectious spondylodiscitis to permanent neurologic outcome was not clear. Patients documented to be bedridden or wheelchair-bound were possibly affected by severe physical de-conditioning during hospitalization rather than a true, significant nerve injury. We could not distinguish these 2 important factors apart. Finally, despite some significant findings, the retrospective study only provides association but not causal relationship.

\section{Conclusion}

This propensity score matched case-control study demonstrated a higher in-hospital and 1-year mortality rate of infectious spondylodiscitis in chronic dialysis patients compared with the non-dialysis control. MRSA comprised the majority of bacterial isolates from the chronic dialysis patients, and the vascular access for hemodialysis contributed to the major focus of bacterial entry. Because of the poorer outcome and high frequency of resistant Staphylococcus, high vigilance, prompt recognition and empiric coverage of MRSA will be important in the management of infectious spondylodiscitis in chronic dialysis patients.

\section{Acknowledgments}

The authors would like to thank Research Services Center for Health Information from Chang Gung Memorial Hospital for statistical analysis (Grant CIRPD1D0031) and Research Grant from Linkou Chang-Gung Memorial Hospital for the support of the maintenance project (Grant CMRPG3F0501 and CORPG3F0111).

\section{Author contributions}

All authors contributed toward data analysis, drafting and revising the paper and agree to be accountable for all aspects of the work.

\section{Disclosure}

The authors report no conflicts of interest in this work.

\section{References}

1. Kehrer M, Pedersen C, Jensen TG, Lassen AT. Increasing incidence of pyogenic spondylodiscitis: A 14-year population-based study. J Infect. 2014;68(4):313-320.

2. Kang SJ, Jang HC, Jung SI, et al. Clinical characteristics and risk factors of pyogenic spondylitis caused by gram-negative bacteria. PLoS One. 2015;10(5):e0127126.

3. Pigrau C, Rodríguez-Pardo D, Fernández-Hidalgo $\mathrm{N}$, et al. Health care associated hematogenous pyogenic vertebral osteomyelitis: a severe and potentially preventable infectious disease. Medicine (Baltimore). 2015; 94(3):e365.

4. Torda AJ, Gottlieb T, Bradbury R. Pyogenic vertebral osteomyelitis: analysis of 20 cases and review. Clin Infect Dis. 1995;20(2):320-328.

5. Chang WS, Ho MW, Lin PC, et al. Clinical characteristics, treatments, and outcomes of hematogenous pyogenic vertebral osteomyelitis, 12-year experience from a tertiary hospital in central Taiwan. J Microbiol Immunol Infect. Epub 2017 Aug 19.

6. Renz N, Haupenthal J, Schuetz MA, Trampuz A. Hematogenous vertebral osteomyelitis associated with intravascular device-associated infections - A retrospective cohort study. Diagn Microbiol Infect Dis. 2017;88(1):75-81.

7. Betjes MG. Immune cell dysfunction and inflammation in end-stage renal disease. Nat Rev Nephrol. 2013;9(5):255-265.

8. Eleftheriadis T, Antoniadi G, Liakopoulos V, Kartsios C, Stefanidis I. Disturbances of acquired immunity in hemodialysis patients. Semin Dial. 2007;20(5):440-451.

9. Hauser AB, Stinghen AE, Kato S, et al. Characteristics and causes of immune dysfunction related to uremia and dialysis. Perit Dial Int. 2008; 28(Suppl 3):S183-S187.

10. Kato S, Chmielewski M, Honda H, et al. Aspects of immune dysfunction in end-stage renal disease. Clin J Am Soc Nephrol. 2008;3(5): $1526-1533$.

11. Abid S, De Silva S, Warwicker P, Farrington K. Infective spondylodiscitis in patients on high-flux hemodialysis and on-line hemodiafiltration. Hemodial Int. 2008;12(4):463-470.

12. Afshar M, Reilly RF. Spondylodiscitis in a patient on chronic hemodialysis. Nat Rev Nephrol. 2011;7(10):599-604.

13. Cervan AM, Colmenero Jde D, Del Arco A, Villanueva F, Guerado E. Spondylodiscitis in patients under haemodyalisis. Int Orthop. 2012; 36(2):421-426.

14. Lu YA, Hsu HH, Kao HK, et al. Infective spondylodiscitis in patients on maintenance hemodialysis: a case series. Ren Fail. 2016;39(1): 179-186.

15. Garcia-Garcia P, Rivero A, del Castillo N, et al. Infectious spondylodiscitis in hemodialysis. Semin Dial. 2010;23(6):619-626.

16. Faria B, Canto Moreira N, Sousa TC, et al. Spondylodiscitis in hemodialysis patients: a case series. Clin Nephrol. 2011;76(5):380-387.

17. Tsuchiya K, Yamaoka K, Tanaka K, Sasaki T. Bacterial spondylodiscitis in the patients with hemodialysis. Spine (Phila Pa 1976). 2004;29(2): 2533-2537.

18. Helewa RM, Embil JM, Boughen CG, et al. Risk factors for infectious spondylodiscitis in patients receiving hemodialysis. Infect Control Hosp Epidemiol. 2008;29(6):567-571.

19. Berbari EF, Kanj SS, Kowalski TJ, et al. Executive summary 2015 Infectious Diseases Society of America (IDSA) clinical practice guidelines for the diagnosis and treatment of native vertebral osteomyelitis in adults. Clin Infect Dis. 2015;61(6):859-863.

20. Sarnak MJ, Jaber BL. Mortality caused by sepsis in patients with endstage renal disease compared with the general population. Kidney Int. 2000;58(4):1758-1764.

21. Sarnak MJ, Jaber BL. Pulmonary infectious mortality among patients with end-stage renal disease. Chest. 2001;120(6):1883-1887. 
22. Durante-Mangoni E, Pafundi PC, Ravasio V, et al. Current features of infective endocarditis in persons on hemodialysis: a prevalence study with case control design from the prospective multicenter SEI cohort. Infection. 2016;44(4):467-474.

23. Abu Sitta E, Habte-Gabr E, Qaraghan Z, Aljariri Alhesan N, RiosBedoya C. Infective endocarditis in haemodialysis patients: lower complications and same mortality rate as in non-haemodialysis patients. Infect Dis (Lond). 2017;49(4):308-311.

24. Bhatia N, Agrawal S, Garg A, et al. Trends and outcomes of infective endocarditis in patients on dialysis. Clin Cardiol. 2017;40(7): 423-429.

25. Tonelli M, Wiebe N, Culleton B, et al. Chronic kidney disease and mortality risk: a systematic review. J Am Soc Nephrol. 2006;17(7):2034-2047.

26. Scott EC, Ho HC, Yu M, Chapital AD, Koss W, Takanishi DM Jr. Pre-existing cardiac disease, troponin I elevation and mortality in patients with severe sepsis and septic shock. Anaesth Intensive Care. 2008;36(1):51-59.

27. Landesberg G, Gilon D, Meroz Y, et al. Diastolic dysfunction and mortality in severe sepsis and septic shock. Eur Heart J. 2012;33(7):895-903.

28. Sapico FL, Montgomerie JZ. Vertebral osteomyelitis. Infect Dis Clin North Am. 1990;4(3):539-550.

29. Nielsen LH, Jensen-Fangel S, Benfield T, et al. Risk and prognosis of Staphylococcus aureus bacteremia among individuals with and without end-stage renal disease: a Danish, population-based cohort study. BMC Infect Dis. 2015;15:6.
30. Skov Dalgaard L, Norgaard M, Jespersen B, et al. Risk and prognosis of bloodstream infections among patients on chronic hemodialysis: a Population-Based Cohort Study. PLoS One. 2015;10(4):e0124547.

31. Danese MD, Griffiths RI, Dylan M, Yu HT, Dubois R, Nissenson AR. Mortality differences among organisms causing septicemia in hemodialysis patients. Hemodial Int. 2006;10(1):56-62.

32. Quarles LD, Rutsky EA, Rostand SG. Staphylococcus aureus bacteremia in patients on chronic hemodialysis. Am J Kidney Dis. 1985;6(6): $412-419$.

33. Zhang J, Burr RA, Sheth HS, Piraino B. Organism-specific bacteremia by hemodialysis access. Clin Nephrol. 2016;86(9):141-146.

34. Romero-Vivas J, Rubio M, Fernandez C, Picazo JJ. Mortality associated with nosocomial bacteremia due to methicillin-resistant Staphylococcus aureus. Clin Infect Dis. 1995;21(6):1417-1423.

35. Selvey LA, Whitby M, Johnson B. Nosocomial methicillin-resistant Staphylococcus aureus bacteremia: is it any worse than nosocomial methicillin-sensitive Staphylococcus aureus bacteremia? Infect Control Hosp Epidemiol. 2000;21(10):645-648.

36. Kim W, Kim SM, Yu H, Jang M, Baek SD, Kim SB. Association between afebrile status and in-hospital mortality among adult chronic hemodialysis patients with bacteremia. Hemodial Int. Epub 2017 Mar 23.
Therapeutics and Clinical Risk Management

\section{Publish your work in this journal}

Therapeutics and Clinical Risk Management is an international, peerreviewed journal of clinical therapeutics and risk management, focusing on concise rapid reporting of clinical studies in all therapeutic areas, outcomes, safety, and programs for the effective, safe, and sustained use of medicines. This journal is indexed on PubMed Central, CAS,

\section{Dovepress}

EMBase, Scopus and the Elsevier Bibliographic databases. The manuscript management system is completely online and includes a very quick and fair peer-review system, which is all easy to use. Visit http://www.dovepress.com/testimonials.php to read real quotes from published authors.

Submit your manuscript here: http://www.dovepress.com/therapeutics-and-clinical-risk-management-journal 